\title{
Eye-movement-driven changes in the perception of auditory space
}

\author{
THÉRÈSE COLlins \\ University of Hamburg, Hamburg, Germany \\ and Paris Descartes University and CNRS, Paris, France \\ AND \\ ToBias HeEd ANd BRIGITTE RöDER \\ University of Hamburg, Hamburg, Germany
}

\begin{abstract}
The perceptual localization of sensory stimuli often depends on body position, and, when action is required, sensory coordinates must be transformed into a motor reference frame. We investigated the role of such a reference frame change on visual and auditory spatial cognition. Participants had to make a saccade to a visual or auditory target and subsequently compare the location of a visual or auditory probe to the remembered location of the target. Neither visual nor auditory localization depended on trial-by-trial variability in saccade endpoint, suggesting that target locations are remapped across saccades in a manner allowing for oculomotor noise. We also compared visual and auditory localization performance before and after the systematic modification of saccade metrics by saccadic adaptation. Adaptation introduced systematic biases into transsaccadic visual and auditory localization behavior. These results show that information about eye movements is taken into account in both visual and auditory spatial cognition. We propose that auditory stimuli are remapped across saccades and that this eye-centered representation contributes to normal auditory localization.
\end{abstract}

The perceptual localization of sensory stimuli is not a straightforward process, because such stimuli are encoded with reference to the body, whose position can change. Different reference frames are used to represent stimuli in different sensory modalities, such as eye centered for visual stimuli and body centered for somatosensory stimuli. Auditory stimuli are initially encoded in a head-centered reference frame; the spatial coordinates of auditory targets are extracted from interaural differences in arrival time and level. The sound emitted by a source is diffracted by its interaction with the head and external ears, and the resulting changes in the temporal characteristics and intensity provide cues about the location of the sound relative to the head (Hofman \& Van Opstal, 1998; Middlebrooks, 1992; Oldfield \& Parker, 1984). When action is required, the representation of sensory stimuli often must be transformed into another reference frame. A visual target encoded in eye-centered coordinates must be transformed into hand-centered coordinates when a manual reach is to be made. Single cell recordings have revealed that audio-guided saccades are coded in an eye-centered reference frame (superior colliculus [SC]: Jay \& Sparks, 1984; frontal eye fields: Russo \& Bruce, 1994; posterior parietal cortex: Stricanne, Andersen, \& Mazzoni, 1996). Saccades to auditory targets are accurate (Frens \& Van Opstal, 1995; Jay \& Sparks, 1984); thus, the preparation of auditory saccades requires a coordinate transforma- tion from head- to eye-centered coordinates. Such transformations are thought to take place in the intermediate and deep layers of the SC (Jay \& Sparks, 1987a, 1987b), where sensory signals about auditory and visual targets converge and are represented in terms of motor error (i.e., the distance between current gaze position and target position; Jay \& Sparks, 1987a).

Eye movements are known to influence the perceived location of visual targets in space (e.g., Collins, DoréMazars, \& Lappe, 2007; Collins, Rolfs, Deubel, \& Cavanagh, 2009; Ross, Morrone, \& Burr, 1997; Ross, Morrone, Goldberg, \& Burr, 2001). In particular, when the metrics of an eye movement are modified by saccadic adaptation, a comparable shift occurs in the perceived location of visual targets (Bahcall \& Kowler, 1999; Collins et al., 2007; Collins et al., 2009). Saccadic adaptation refers to the remarkable ability of the brain to adjust the motor parameters of eye movements relative to the sensory coordinates of the target. Adaptation can be observed after impairment of the extraocular muscles or nerve lesions: Initially ill-directed saccades gradually adjust, and after a few days are once again appropriate to the target (Abel, Schmidt, Dell'Osso, \& Daroff, 1978; Kommerell, Olivier, \& Theopold, 1976; Optican \& Robinson, 1980). In these cases, saccadic adaptation realigns saccade metrics onto sensory coordinates. In the laboratory, displacing the saccade target during movement execution introduces

T. Collins, collins.th@gmail.com 
an artificial targeting error, because visual perception is reduced during eye movements and the target step goes unnoticed (Bridgeman, Hendry, \& Stark, 1975). However, there is a progressive modification of saccadic amplitude such that the amplitude eventually becomes appropriate to the postsaccadic target position (McLaughlin, 1967). In this case, adaptation can be seen as dissociating the motor properties of the saccade from the sensory coordinates of the target. Importantly, the locations of visual objects present around the time of the saccade are also misjudged: The perceived locations of visual probes shift in the direction of the saccadic adaptation (Bahcall \& Kowler, 1999; Collins et al., 2009). The change in the motor characteristics of the saccade seems to modify the perceived spatial characteristics of visual objects.

The present study examined whether saccade metrics are involved in the perceived location of auditory targets. We examined two sources of saccade metric variability: habitual trial-by-trial variability and amplitude changes due to saccadic adaptation. Participants had to saccade to auditory targets and subsequently compare the location of an auditory probe to that of the target. We also ran a visual version of the task to be able to directly relate the results of our new, auditory task to previous work on visual localization. We hypothesized that, because sensory information about both auditory and visual stimuli are represented in eye-centered coordinates in the SC and because saccadic adaptation takes place at this or a lower level (such as the cerebellum; Hopp \& Fuchs, 2004), the localization of auditory stimuli would shift following the adaptive modification of saccade metrics.

\section{METHOD}

\section{Participants}

Eighteen participants (ages: $21-38 ; 7$ women) from the University of Hamburg community took part in the experiment in exchange for payment or course credit. All participated in the auditory localization session, and 6 participated in both the auditory- and visuallocalization sessions (see below). All were naive with regard to the object of the experiment (except one author, T.C.), had normal visual acuity, and had no known auditory deficits or neurological disorders. All gave their informed consent prior to starting the experiment, which was carried out according to the ethical standards of the Declaration of Helsinki (2004).

\section{Instruments and Stimuli}

Visual stimuli consisted of $1^{\circ}$-diameter dots presented on an Ilyama MS103DT 21-in. screen (vertical refresh rate, $170 \mathrm{~Hz}$ ). Participants sat $57 \mathrm{~cm}$ from the screen, and their heads were kept stable by chin and forehead rests. Auditory stimuli were presented via Oticon bone conductors mounted on foam fixed to the screen at $2.5^{\circ}$ center-to-center intervals (Figure 1). A sound stimulus consisted of a 30-msec tone emitted from one of the bone conductors at $167 \mathrm{~Hz}$ (i.e., one cycle was $6 \mathrm{msec}$ long). To counteract small variations in the stimulators' sound characteristics, we randomly varied the time the stimulator was turned on during each cycle (on-time $0.01-0.1 \mathrm{msec}$ in $0.01-\mathrm{msec}$ steps). This manipulation varied the loudness of the stimulators from 50 to $56 \mathrm{~dB}$ (measured at the location of the ears during the experiment) and prevented participants from determining the location of the auditory stimuli on the basis of small differences between stimulators. The stimulators were covered with acoustically transparent speaker fabric that prevented participants from seeing the location of each sound source.

Eye movements were monitored with an Eyelink 1000 Remote (SR Research, Osgoode, ON) at a 500-Hz sampling rate. Saccades were detected online on the basis of velocity $(>30 \% \mathrm{sec})$ and acceleration $\left(>3,000^{\circ} / \mathrm{sec}^{2}\right)$ thresholds. At the beginning of a session, the

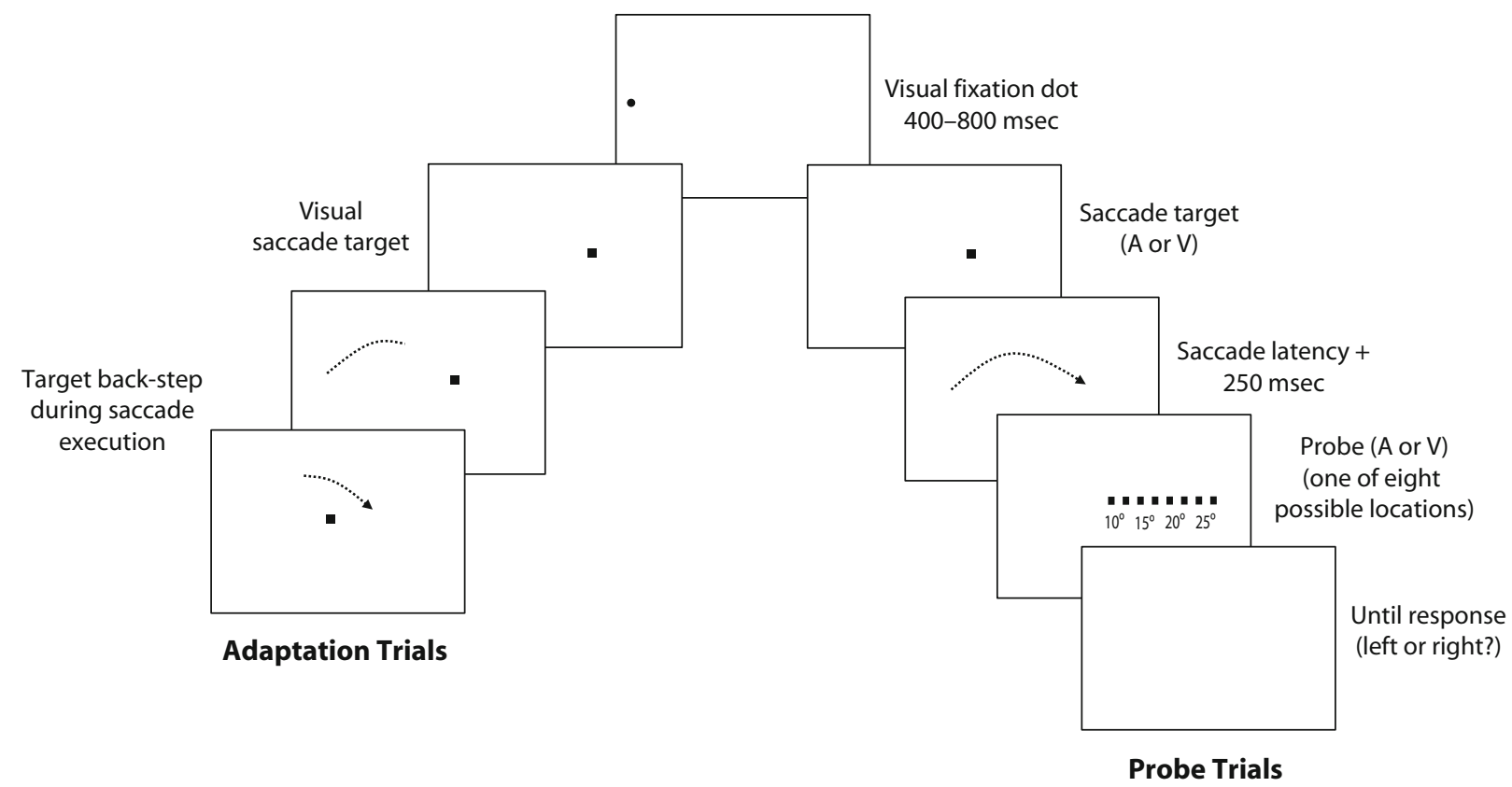

Figure 1. Procedure. Participants fixated the visual fixation dot and pressed a button. After a random interval of $400-800 \mathrm{msec}$, the saccade target appeared. Participants were instructed to make a saccade to the target as soon as it appeared. The screen remained blank for $250 \mathrm{msec}$ after saccade detection, and then the probe was presented at one of eight locations (between $10^{\circ}$ and $27.5^{\circ}$ in $2.5^{\circ}$ steps). In the auditory localization task, the auditory target was presented for $60 \mathrm{msec}$. In the visual localization task, the visual saccade target was presented until saccade detection. 
Eyelink was calibrated. Just before each trial, central fixation was checked and compared with the calibration. If the distance between the fixation check and the calibration was greater than $1^{\circ}$, fixation was refused and a full calibration was initiated. Eye-movement traces were later analyzed offline. Instantaneous velocity and acceleration were computed for each data sample and were compared with a threshold $\left(30^{\circ} / \mathrm{sec}\right.$ and $\left.8,000^{\circ} / \mathrm{sec}^{2}\right)$. Saccade onset was defined as two consecutive above-threshold samples for both criteria. Saccade offset was defined as the beginning of the next $20-\mathrm{msec}$ period of below-threshold samples.

\section{Procedure}

We ran two experimental sessions: one with an auditory localization task and one with a visual localization task. Each session was divided into three successive phases. In the preadaptation phase, we recorded normal saccades to auditory (or visual) targets, and participants judged the location of auditory (or visual) probes. Then saccades were adapted visually: Participants had to saccade to a visual target that stepped back in the direction of fixation during saccade execution. In the postadaptation phase, we again recorded saccades to auditory (or visual) targets, and participants judged the location of auditory (or visual) probes.

Visual localization task. Participants $(n=6)$ fixated a black fixation dot located $12^{\circ}$ to the left of screen center and pressed on a button when they were ready. The buttonpress initiated a fixation check. If fixation was comparable to the calibration values, the dot turned gray. After a random fixation duration of $400-800 \mathrm{msec}$, a visual target $\left(1^{\circ}\right.$-diameter gray dot) located $20^{\circ}$ to the right of fixation was presented simultaneous to fixation dot offset. Participants were instructed to make a saccade to the target as accurately as possible and to maintain fixation on the new position. The target disappeared upon saccade detection; $250 \mathrm{msec}$ later, a visual probe appeared (also a $1^{\circ}$-diameter gray dot), chosen randomly from one of eight locations (Figure 1). By pressing one of two buttons at their right hand, participants indicated whether the probe was to the left or right of the saccade target (two-alternative forced choice paradigm). Each experimental session was composed of 160 preadaptation visual localization trials ( 20 per probe location), followed by 140 saccadic adaptation trials. The postadaptation trials were either visual probe trials $(n=160)$ or saccadic adaptation trials $(n=40)$, during which a visual target was stepped back during the saccade (see below). Saccadic adaptation trials were included to maintain adaptation throughout the postadaptation phase.

Auditory localization task. The procedure was similar to the visual localization task, except that auditory stimuli were used as targets and probes. Participants $(n=18)$ fixated the fixation dot. If the fixation check was satisfactory, after a random fixation duration of $400-800 \mathrm{msec}$, a sound source located $20^{\circ}$ to the right of fixation was presented for $60 \mathrm{msec}$, simultaneous to fixation dot offset. Two hundred fifty msec after the saccade landed, the sound source (the probe) reappeared for $60 \mathrm{msec}$, located randomly at one of the eight possible locations (Figures 1A-1B). Participants indicated whether the probe was to the left or right of the saccade target. Each experimental session was composed of 240 preadaptation auditory localization trials ( 30 per probe location), 140 saccadic adaptation trials, and finally 320 postadaptation trials (240 auditory probe trials and 80 adaptation trials).

Saccadic adaptation. Amplitude-reducing adaptation was evoked classically by stepping the visual saccade target $5^{\circ}$ in the direction opposite to the saccade during its execution. Participants fixated the fixation dot and made a saccade to the $20^{\circ}$ visual target as soon as it appeared (simultaneously with fixation dot offset). During saccade execution, the dot was stepped back by $5^{\circ}$ in the direction of fixation (Figure 1). No sound sources were used during adaptation trials.

\section{Data Analyses}

Average saccade latency and amplitude were analyzed with a twoway ANOVA, including factors phase (preadaptation vs. postadapta- tion) and probe location (eight locations; shown in Figure 1). When Mauchly's test was significant and sphericity could not be assumed, we used Greenhouse-Geisser adjustments to the degrees of freedom (although, uncorrected $d f \mathrm{~s}$ are reported in the Results section). The amount of saccadic adaptation in percentage was calculated as [(pretest saccade amplitude - posttest saccade amplitude)/ pretest saccade amplitude]. Full saccadic adaptation would be $25 \%$ $[(20-15) / 20]$. A logistic psychometric function for the perceptual report (probe occurred left vs. right) was fitted for each participant's data for the pre- and postadaptation phases. We defined the perceptual null location (PNL, that location at which a participant judged the probe location to be identical to the saccade target location) to be the point of this curve at which the probability of both a "right" and a "left" response was $50 \%$. PNLs for each phase were compared with a Student $t$ test for dependent measures.

\section{RESULTS}

\section{Visual Localization}

Saccade characteristics. Average saccade latency was $157 \pm 13 \mathrm{msec}$ (average $\pm S E M$ ). There was a small difference between pre- and postadaptation phases, probably due to practice $[171 \pm 12 \mathrm{msec}$ and $143 \pm 11 \mathrm{msec}$, respectively; $F(1,5)=21.7, p<.007]$. As we expected, there was no influence of probe location, because probes appeared after saccade landing $[F(7,35)=1.5, p>.25]$, and there was no interaction between the two factors $[F(7,35)=2.3, p>.11]$.

Average saccade amplitude reduced from $17.2^{\circ} \pm 0.6^{\circ}$ to $14.9^{\circ} \pm 0.4^{\circ}$ between pre- and postadaptation phases $[F(1,5)=56.0, p<.001]$, with no effect of probe location $[F(7,35)=2.0, p>.17]$ and no interaction $[F(7,35)=1.3, p>.30]$. Average saccadic adaptation was $13 \% \pm 4 \%$ (range: $6 \%-17 \%$ ). The target back-step was $5^{\circ}$ (from $20^{\circ}$ to $15^{\circ}$ ), and the difference between preadaptation and postadaptation amplitudes was $2.3^{\circ} \pm 0.3^{\circ}$, which corresponds to a $46 \% \pm 6 \%$ compensation of the $5^{\circ}$ back-step. Figure 2 presents the time course of adaptation in a typical participant, and Figure 3 presents the amplitude change between pre- and postadaptation phases for all participants.

Visual probe localization. The average PNL corresponds to the location at which the visual probe was perceived to be equal to that of the visual saccade target. Before adaptation, average PNL was $0.35^{\circ} \pm 0.42^{\circ}$ (distance measured from the location of the saccade target), which corresponds to veridical localization (not significantly different from $0, t<1$ ). After adaptation, average PNL decreased to $-2.1^{\circ} \pm 0.7^{\circ}[t(5)=3.62, p<.01]$; see Figure 4A. After saccadic adaptation, the psychometric function shifted leftward, indicating that probes presented to the left of the target location were perceived at the same location, whereas probes presented at the same location as the target were judged to be to the right (Figure 4B).

The size of the perceptual shift was comparable to the amount of adaptation (change of $2.2^{\circ} \pm 0.5^{\circ}$ vs. change of $2.3^{\circ} \pm 0.3^{\circ}$ ), but there was no correlation between the amount of adaptation and the perceptual report (i.e., $\Delta$ PNL; Pearson's $r=-.22, p>.6$ ); see Figure 5A. We examined whether trial-by-trial variations in eye position not due to saccadic adaptation contributed to localization 


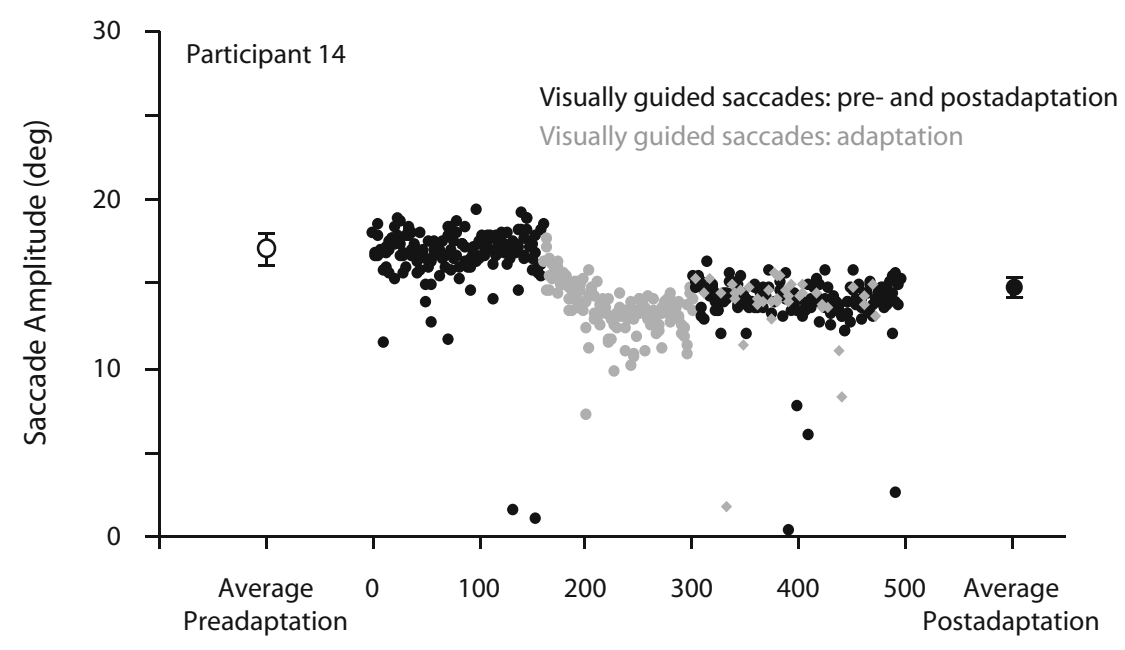

Trial Number

Figure 2. Time course of adaptation in 1 participant in the visual localization task. Each point represents one saccade in preadaptation (black), adaptation (gray), and postadaptation (gray, adaptation trials; black, probe trials) sessions. The average of the 6 participants is given for pretest and posttest sessions with $S E M$.

\section{Participant}

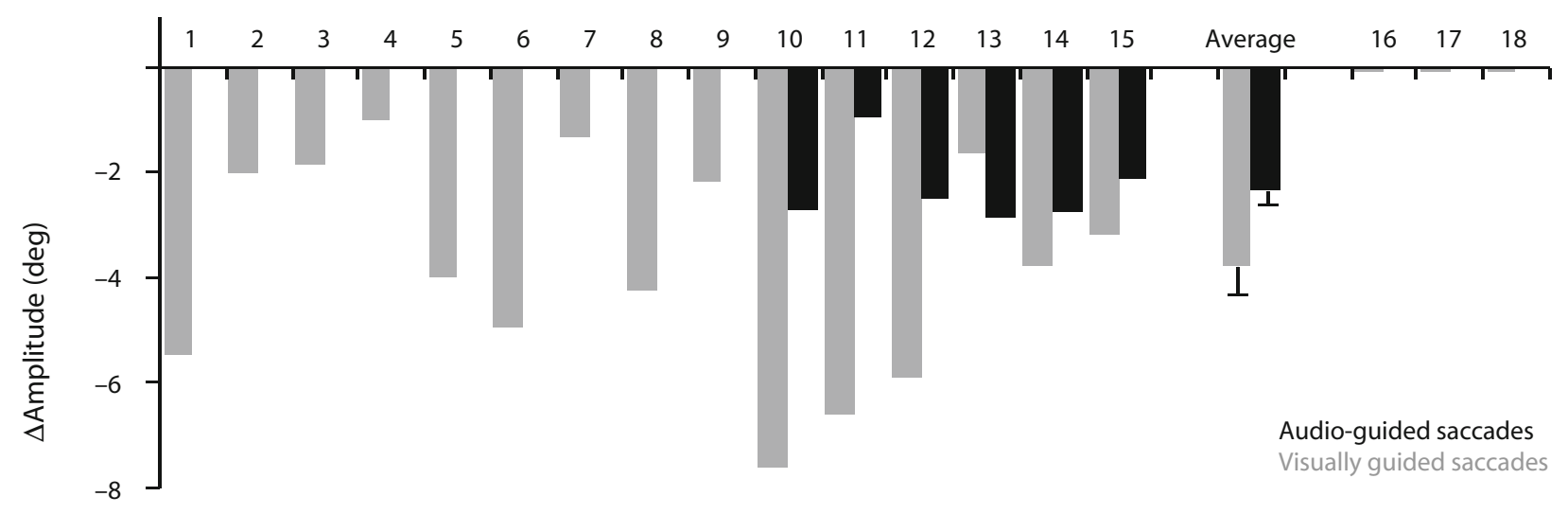

Figure 3. Change in saccade amplitude between pre- and postadaptation phases for each participant, showing average and SEM. To represent the shortening of amplitude, the change is plotted as negative. Saccades recorded in the auditory localization task are plotted in gray and correspond to the transfer of visual adaptation to audio-guided saccades. Saccades recorded in the visual localization task are in black and correspond to the transfer of visual adaptation to visually guided saccades. Note that only Participants 10-15 performed both the visual and auditory tasks. Results for Participants 16, 17, and 18 are separated to the right, because they showed no transfer of visual adaptation to audio-guided saccades and were not included in the averaged results (see text). Their change of

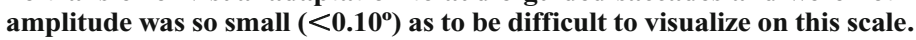

performance. To do so, we analyzed saccade error - the distance between saccade endpoint and saccade target. We divided trials into $1^{\circ}$ bins of saccade error and calculated the percentage of rightward responses (for all probes) for each bin. We analyzed pre- and postadaptation phases independently. There was no systematic relationship between saccade error and perceptual report: Spearman's correlation coefficients were nonsignificant (all $p \mathrm{~s}>.30$ ) for both phases (Figure 5B). These flat functions reveal that the perceived location of the visual probe did not depend on the actual saccade endpoint.
Auditory localization. A prerequisite for examining the effect of saccade metrics on auditory localization was the successful transfer of visual saccadic adaptation to auditory saccades. Without an amplitude difference between the saccades aiming for the auditory target in pre- versus postadaptation phases, we could not compare auditory localization between the two sessions to test our hypotheses. As shown in Figures 3 and 4, Participants 16, 17, and 18 did not show transfer of visual saccadic adaptation to auditory saccades, despite the apparent adaptation of visually guided saccades in the adaptation phase (the 
A Participant

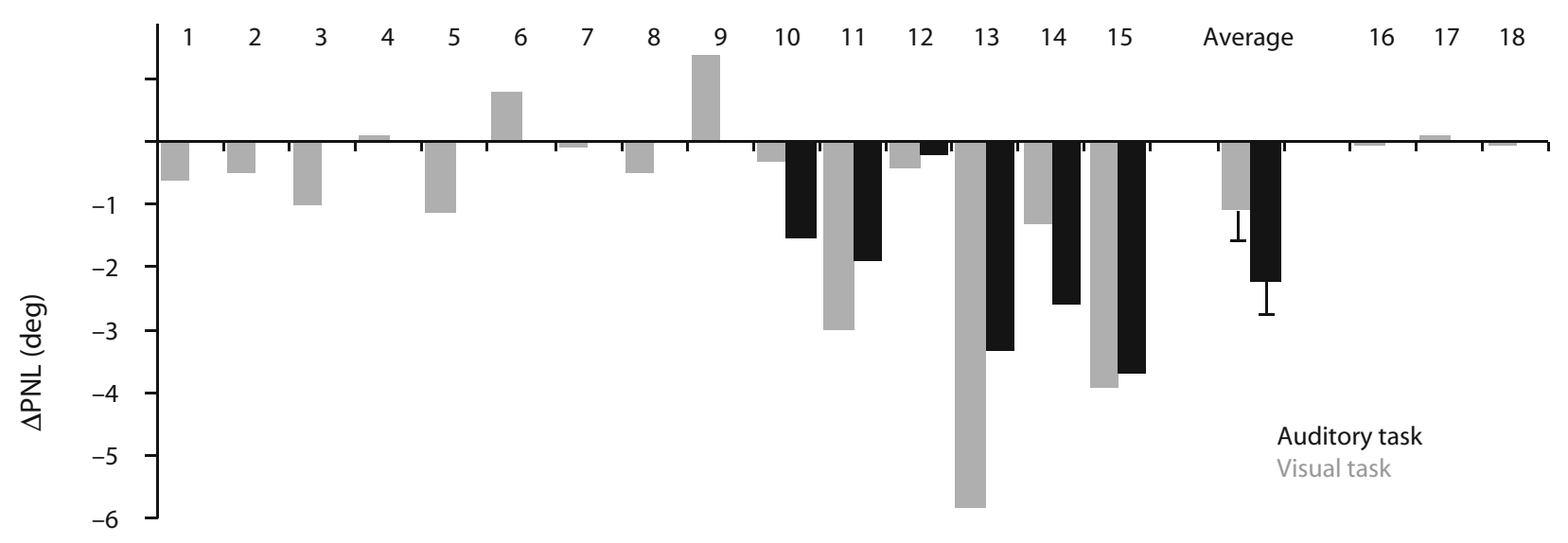

B

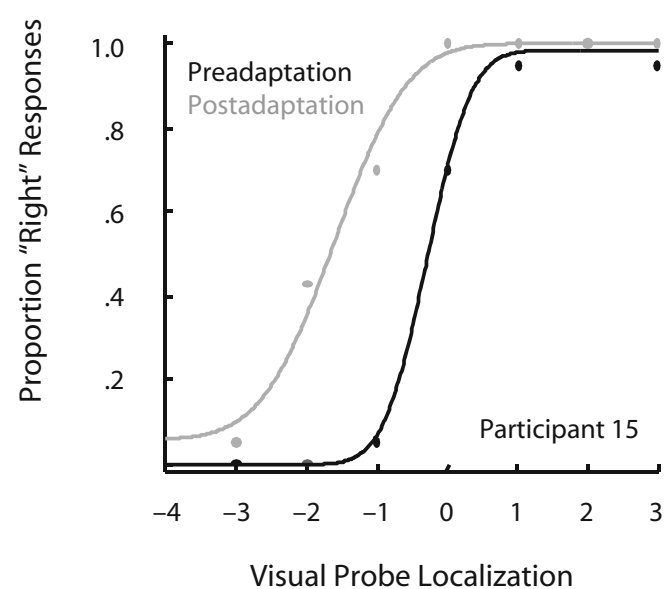

C

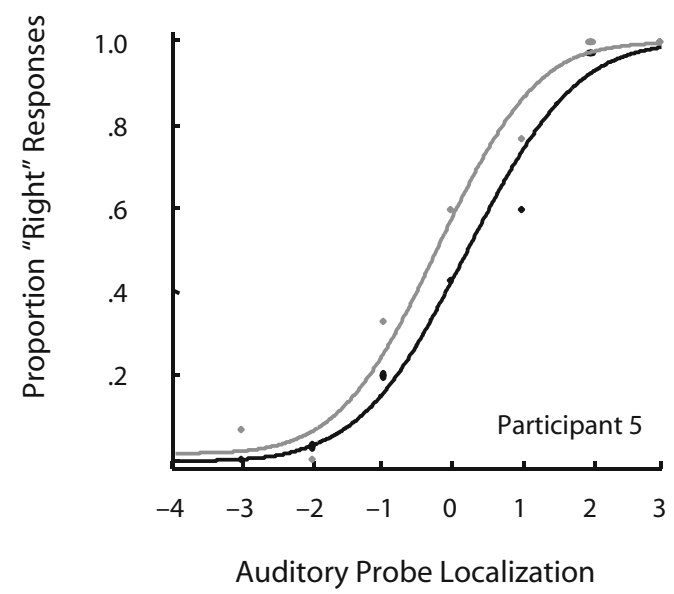

Figure 4. (A) Change in perceptual null location (PNL) between pre- and postadaptation phases for each participant, plus average and $S E M$. To represent the shortening of amplitude, the change is plotted as negative. The results for Participants 16,17 , and 18 are difficult to visualize on this scale because they were so small. (B) Example psychometric functions from 1 participant in the visual localization task in both preadaptation (black) and postadaptation (gray) sessions. (C) Example psychometric functions from 1 participant in the auditory localization task.

average amplitude difference between the first and last 25 trials of the adaptation phase in these 3 participants was $\left.2.6^{\circ} \pm 0.6^{\circ}\right)$. These participants were removed from the average analyses. The following analyses take the 15 remaining participants into account.

Saccade characteristics. Average saccade latency was $191 \pm 11 \mathrm{msec}$. Practice probably led to the difference between pre- and postadaptation $[208 \pm 12 \mathrm{msec}$ and $175 \pm 9$ msec, respectively; $F(1,14)=14.7, p<.003]$, but there was no influence of probe location $[F(7,98)=1.3$, $p>.22]$ and no interaction $(F<1)$.

Average saccade amplitude to auditory targets was $17.1^{\circ} \pm 0.9^{\circ}$ in the pretest and reduced to $13.4^{\circ} \pm 0.7^{\circ}$ in the posttest $[F(1,14)=48.8, p<.001]$. Probe location and the interaction of phase $\times$ probe location were not significant $(p s>.18)$. Figure 6 presents a typical time course of adaptation in an individual participant, and Figure 3 shows the change in amplitude between pre- and postadaptation phases for all 15 participants. Average saccadic adaptation was $21 \% \pm 3 \%$ (range: $8 \%-40 \%$ ), and the difference between preadaptation and postadaptation amplitudes was $3.7^{\circ} \pm 0.5^{\circ}$, which corresponds to a $74 \% \pm 11 \%$ compensation of the $5^{\circ}$ back-step.

Comparison of Figures 2 and 6 suggests that the scatter of auditory saccades is greater than that of visually guided saccades. Indeed, within-participant standard deviations were, on average, $2.5^{\circ}$ and $3.9^{\circ}$ for visually guided and auditory saccades, respectively (such a difference has been observed before; Frens \& van Opstal, 1994, 1995).

Auditory probe localization. The average pretest PNL was $0.16^{\circ} \pm 0.4^{\circ}$, which means that participants' auditory localization judgments were accurate (not significantly different from $0, t<1$ ). The psychometric function shifted after saccadic adaptation, and the postadaptation 


\section{A Saccadic Adaptation (\%)}

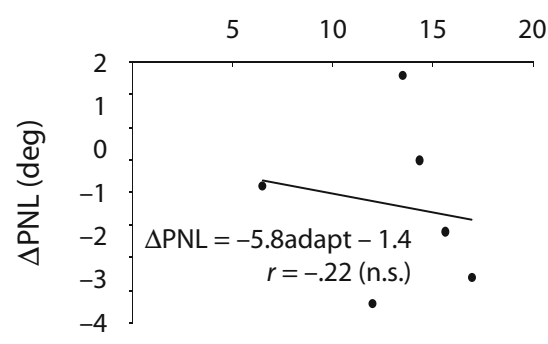

\section{B}

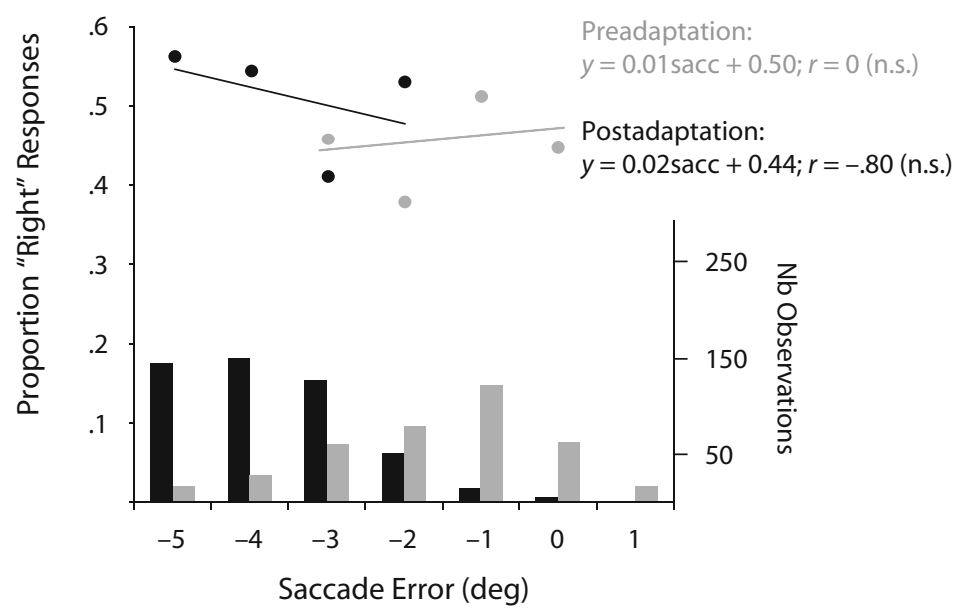

Figure 5. (A) Correlation between the change in perceptual null location (PNL) in the visual localization task and the amount of adaptation. (B) Rightward responses for all probes in the visual localization task as a function of saccade error in preadaptation (gray) and postadaptation (black) phases.

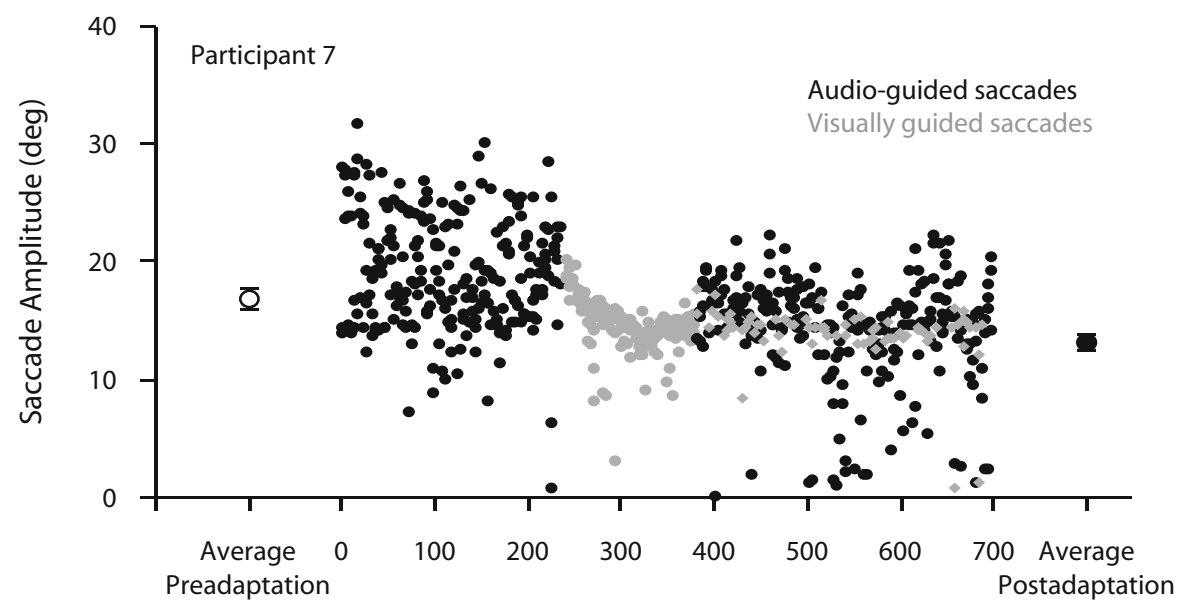

Trial Number

Figure 6. Time course of adaptation in 1 participant in the auditory localization task. Each point represents one saccade in preadaptation (black), adaptation (gray), and postadaptation (gray, adaptation trials; black, probe trials) sessions. 


\section{A Saccadic Adaptation (\%)}

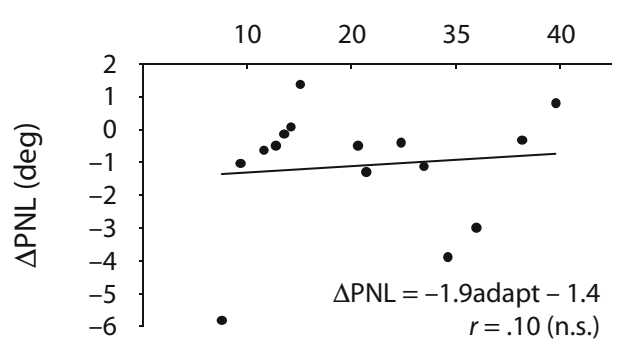

B

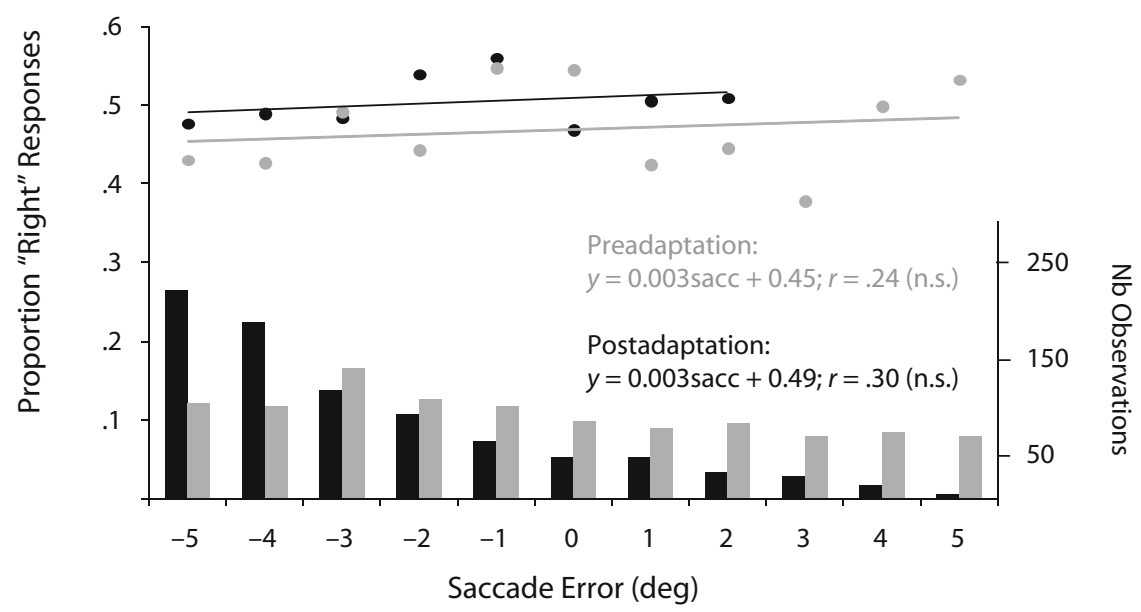

Figure 7. (A) Correlation between the change in perceptual null location (PNL) in the auditory localization task and the amount of adaptation. (B) Rightward responses for all probes in the auditory localization task as a function of saccade error in preadaptation (gray) and postadaptation (black) phases.

PNL was significantly smaller than that of the preadaptation phase $\left[-0.92^{\circ} \pm 0.6^{\circ} ; t(14)=2.26, p<.02\right]$; see Figures 4A and 4C. On the single-participant level, 12 of 15 participants showed this effect numerically. This shift reveals that, after saccadic adaptation, probes presented to the left of the target location were perceived as occupying the same location, whereas probes presented at the same location as the target were perceived as located to the right.

The size of the perceptual shift was smaller than the amount of adaptation (change of $1.08^{\circ} \pm 0.48^{\circ}$ vs. change of $\left.3.7^{\circ} \pm 0.5^{\circ}\right)$. The amount of adaptation was uncorrelated with the size of the perceptual shift (Pearson's $r=.10$, $p>.7$ ); see Figure 7A. As for the visual localization task, by comparing saccade error and perceptual report, we also examined whether trial-by-trial variations in eye position contributed to localization performance. There was no systematic relationship between saccade error and perceptual report, with nonsignificant (all $p \mathrm{~s}>.20$ ) Spearman's coefficients for both phases (Figure 7B). These flat functions reveal that the perceived location of the auditory probe did not depend on the actual saccade endpoint.

Figures 3 and 4 show the results for Participants 16, 17, and 18 , who did not show a difference in auditory sac- cade amplitude between preadaptation and postadaptation phases and whose results were not included in the above analyses. Figure 3 confirms the absence of an amplitude change: $\left(<0.1^{\circ}\right.$ change in all 3 participants); Figure 4 shows that they also had no change of PNL in the postadaptation (differences between pre- and postadaptation were $-0.06,0.09$, and -0.06 for the 3 participants, respectively).

We hypothesized that there would be a positive correlation between the PNL shift in the visual localization task and the PNL shift in the auditory localization task. The absence of a correlation between the amount of adaptation and the perceptual shift suggests that two independent mechanisms might be at work - one responsible for saccade amplitude adaptation and the other responsible for perceptual shifts. However, it is also likely that the two mechanisms are outputs of a common source, but that this is masked by uncorrelated noise (as suggested by the absence of adaptation, accompanied by an absence of PNL shift in 3 participants). We compared the PNL shifts in each task for the 6 participants who performed both the visual and the auditory tasks. Figure 8 shows that the correlation was high and significant (Pearson's $r=.78$, onetailed $p<.035$ ). 


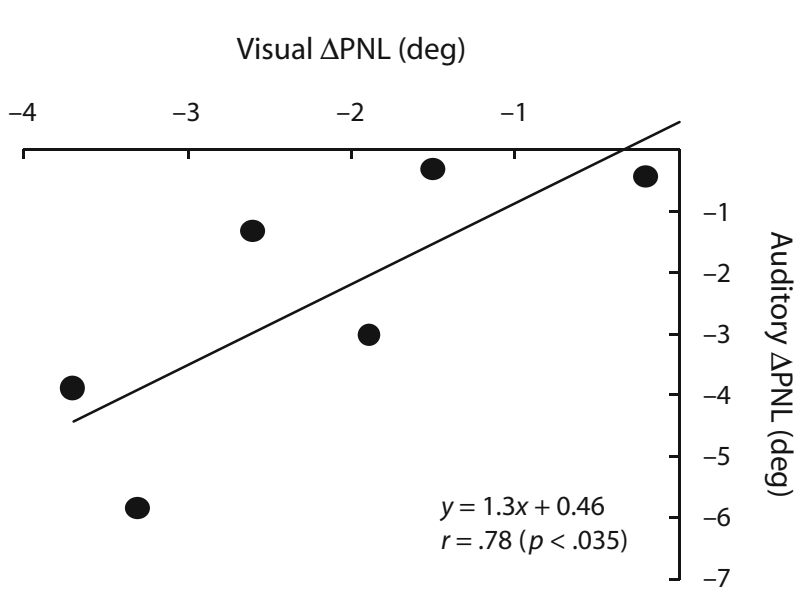

Figure 8. Correlation between perceptual null location (PNL) in the visual and auditory localization tasks. Each point represents 1 participant.

\section{DISCUSSION}

The goal of the present study was to examine whether saccade metrics influenced auditory localization. Sounds are initially encoded in a head-centered reference frame but must be transformed into an eye-centered reference frame in order for a saccade to be made toward the sound source. The transformation from head-centered to eyecentered coordinates is thought to occur at the level of the SC (Jay \& Sparks, 1987a, 1987b), and we therefore hypothesized that saccadic adaptation, thought to involve this or a lower level (Hopp \& Fuchs, 2004), may influence auditory localization.

We first ran a visual localization task to assess the influence of eye movements and adaptation on perceived visual location. This task was included to replicate previous work and to enable us to compare our visual and auditory tasks directly. Our results show that visual localization does not depend on normal oculomotor variability, replicating previous work (Collins et al., 2009). There was no correlation between the actual saccade endpoint and perceptual reports of visual localization. Small changes in object position across saccades tend to go unnoticed (Bridgeman et al., 1975) except when the target is absent at the saccade landing and reappears a few hundred milliseconds later (Deubel, Bridgeman, \& Schneider, 1998). Our paradigm included such a gap, and we therefore expected, and observed, good localization performance in the preadaptation phase. Such good performance suggests that there is a precise spatial map for visual objects. The presaccadic target would be encoded on this map and remapped after the saccade to its expected postsaccadic coordinates (Melcher \& Colby, 2008). Such a remapping could take the form of a vector subtraction between the presaccadic target coordinates and the upcoming saccade vector. The postsaccadic probe would then be compared with the remapped target coordinates, and a judgment about whether the postsaccadic probe was to the left or right could be made (Collins et al., 2009; Deubel, 2004). However, a certain amount of noise is associated with the remapping process and also with actual saccade amplitude. Thus, we hypothesized that perceptual responses might depend on the actual saccade endpoint, which could introduce artificial discrepancies between the remapped target coordinates and the actual probe coordinates. If this were the case, we would have expected, for example, slightly more rightward responses for probes at the same location as the presaccadic target when the saccade landed to the left of the target location. We did not observe such a dependence of perceptual report on actual saccade endpoint: Small, normal variations in saccade endpoint were discounted by perception, suggesting that the perceptual system ignores habitual saccadic variability (Collins et al., 2009). In contrast, saccadic adaptation shifted the perceived location of visual objects: When the postsaccadic probe and presaccadic target were at the same location, the probe was nevertheless often judged to be to the right of the presaccadic target, whereas probes actually located to the left were often judged to be at the same location as the presaccadic target. This effect of saccadic adaptation on visual localization suggests that the remapped postsaccadic target coordinates are based on an adapted saccade vector (Doré-Mazars, Vergilino-Perez, Collins, Bohacova, \& Beauvillain, 2006), corresponding to a modified localization of the presaccadic target (Awater, Burr, Lappe, Morrone, \& Goldberg, 2005; Bruno \& Morrone, 2007; Collins et al., 2007).

Because each eye movement changes the retinal location of visual objects, and because visual perception is suppressed during saccades, much research has been devoted to transsaccadic visual perception (see Melcher \& Colby, 2008, for a review). In the present study, we examined whether intervening eye movements influenced auditory localization. Acoustic stimuli are encoded in a head-centered reference frame based on cues at the ears-interaural intensity differences, timing differences, and spectral cues (Blauert, 1997). Eye movements do not change head-centered representations, so such movements could theoretically be ignored by the auditory system. There is evidence, however, that auditory stimuli are encoded in an eye-centered reference frame at multiple cortical levels (Groh, Trause, Underhill, Clark, \& Inati, 2001; Jay \& Sparks, 1984, 1987a; Russo \& Bruce, 1994; Stricanne et al., 1996; Werner-Reiss, Kelly, Trause, Underhill, \& Groh, 2003) or in a hybrid head- and eyecentered reference frame (Mullette-Gillman, Cohen, \& Groh, 2005, 2009). Thus, according to the remapping scheme proposed above, intervening eye movements could influence auditory localization.

Our results showed that, as is the case with visual localization, perceptual reports for auditory localization did not covary with the normal-variability actual saccade endpoint. If auditory localization does take saccade metrics into account, it discounts normal oculomotor variability. These results could also suggest that auditory localization is not at all influenced by eye movement metrics. Indeed, the probe localization task could be solved by using headcentered coordinates only: A participant could ignore whatever eye movement she makes and simply compare the two head-centered coordinates of the targets. We show 
that this is not the case, because the shift of localization in the direction of the adapted saccade metrics suggests that eye movement signals are used for auditory localization even in the absence of head movements. We measured auditory localization before and after saccadic adaptation obtained with visually guided saccades transferred to saccades of similar amplitude aiming for an auditory target. Such cross-modal transfer of adaptation has been reported before (Frens \& van Opstal, 1994, 1995). The new finding in the present study is that this change of the motor plan of saccades to auditory targets also influences the perceptual localization. Localization was veridical before adaptation, but shifted after adaptation, in such a way that auditory probes presented at the same location as the presaccadic target were often perceived to be located farther away than the presaccadic target, whereas probes presented closer to the original fixation position than the presaccadic target were often perceived to occupy the same position.

Three of our participants, despite apparent adaptation of visually guided saccades, did not show a transfer of adaptation to audio-guided saccades. They also did not show a perceptual effect, and probe localization judgments were similar before and after adaptation. Although these are null results, they nevertheless strengthen our proposal that the shift in auditory PNL was tied to the modification of saccade metrics.

Our results are compatible with several reports of static eye position influences on responses to auditory stimuli (Lackner, 1973; Lewald, 1997, 1998; Lewald \& Ehrenstein, 1996a, 1996b; Weerts \& Thirlow, 1971). These studies showed small but consistent shifts of perceived sound location in the direction opposite to that of orbital eye deviation (i.e., deviation of the eyeball within the orbit to one position or another) in the order of $2^{\circ}$ of azimuth. In other words, when the eyes fixated a position in the left visual field, sounds were localized as more to the right than they actually were. However, the effect of eye deviation could be the result of the modification of the reference point required for the judgment by the eccentric eye fixation (e.g., the subjective midline; see Lewald \& Ehrenstein, 2000). Our results also show an effect of eye movements on auditory localization in the direction opposite to that of the eye movement: Adapted rightward saccades were accompanied by a leftward shift in perceived auditory location. This might suggest that the mechanisms for judging auditory location during static fixation and across eye movements are functionally related; however, we did not directly compare static eye position with transsaccadic auditory localization and so cannot draw any conclusions about this point. Goossens and Van Opstal (1999) and Vliegen, Van Grootel, and Van Opstal (2004) also examined the influence of intervening eye movements on auditory localization. Their participants performed a combined eye-head movement to an auditory stimulus following an eye-head movement to a visual stimulus. Localization was indexed by the accuracy of the eye-head movement to the auditory stimulus. The motor response was accurate, and the intervening eye-head movement was compensated for, suggesting that sound localization was stably represented across eye-head movements. Note, however, that the re- sponse was a motor task, and dissociations of perceptual versus action responses have been shown for visual localization (Burr, Morrone, \& Ross, 2001). Kopinska and Harris (2003) examined auditory localization with a perceptual task and showed that there was no effect of intervening eye movements on auditory localization. However, the sounds in their study were presented intracranially through headphones. As noted by Pavani, Husain, and Driver (2008), an influence of eye movements on auditory localization might be expected for free-field auditory stimuli, because realignment of auditory and visual maps after intervening eye movements is probably more relevant for keeping the multisensory integrity of real objects occurring in the outside world than for matching artificial sounds heard intracranially to visual targets (Pavani et al., 2008; Pavani, Husain, Làdavas, \& Driver, 2004). Pavani et al. (2008) used a perceptual task with free-field auditory stimuli, in which participants reported whether the location of an acoustic stimulus presented after an eye movement was different from that of an acoustic stimulus presented before the eye movement. Sensitivity for the location discrimination selectively dropped when the second sound shifted in the direction opposite to the intervening eye movement, suggesting that the first sound was represented (and updated) in eye-centered coordinates. Our study differs from theirs in that we required participants to perform only one $15^{\circ}-20^{\circ}$ saccade, whereas in the Pavani et al. (2008) study, participants had to change fixation location between two peripheral locations $50^{\circ}$ apart, which was often done with more than one saccade. We were therefore able to examine the influence of saccade metrics rather than just saccade occurrence.

What could the physiological underpinnings of the effect of saccadic adaptation on auditory localization be? There is evidence that auditory stimuli are encoded in an eye-centered reference frame at several cortical and subcortical structures. However, for several reasons, the $\mathrm{SC}$ is a probable candidate as the mediator of these adaptation effects. Modified neuronal activity after adaptation has been reported in the SC of macaque monkeys (Takeichi, Kaneko, \& Fuchs, 2007). The involvement of the SC in adaptation is also supported by behavioral data investigating the characteristics of the transfer of adaptation from a given saccade to other saccades (Hopp \& Fuchs, 2002; see Hopp \& Fuchs, 2004, for a review). These findings support the idea that the SC might be the site of adaptation or that adaptation is present in collicular inputs. Furthermore, observations that lesions of the cerebellar thalamus impair adaptation in humans (Gaymard, Rivaud-Péchoux, Yelnik, Pidoux, \& Ploner, 2001) and observations of specific adaptation deficits in Parkinson's patients (MacAskill, Anderson, \& Jones, 2002) corroborate this hypothesis. Finally, remapping of sensory targets across saccades requires information about the metrics of the upcoming saccade. Signals carrying such information, called efference copy signals, or corollary discharge signals, have been identified in the SC (Sommer \& Wurtz, 2008). Given these convergent findings, current models of saccadic adaptation place its site at the level of the SC. 
Although the shift in visual localization was comparable to that of the adaptive saccade amplitude reduction, the size of the shift in auditory localization was smaller than that of the motor change. This difference may stem from the fact that auditory localization relies on several mechanisms, including contributions from areas coding auditory targets in eye-centered coordinates, as well as from structures using head-centered coordinates whose function would not be affected by saccadic adaptation. The shift of auditory localization following saccadic adaptation is reminiscent of the ventriloquism aftereffect, an enduring shift of the perceived spatial location of auditory stimuli after exposure to spatially disparate but simultaneous acoustic and visual stimuli (Lewald, 2002; Recanzone, 1998). The ventriloquism aftereffect is a kind of cross-modal spatial adaptation, and, similar to the results in the present study, the perceptual effect is smaller than the adapting disparity.

In summary, we showed that visual localization does not vary with trial-by-trial variability in saccade endpoint, suggesting that visual perception discounts normal oculomotor noise. Visual target locations are remapped across saccades in a manner allowing for oculomotor noise. Saccadic adaptation disrupts this remapping and introduces systematic biases into visual localization behavior. Transsaccadic auditory localization shows similar effects. Trial-by-trial oculomotor variability did not influence perceived auditory location, but systematic biases in localization behavior were observed after saccadic adaptation. We propose that auditory stimuli, like visual stimuli, are remapped across saccades and that this remapped eye-centered representation contributes to normal auditory localization.

\section{AUTHOR NOTE}

T.C. was supported by an Alexander von Humboldt Research Fellowship. Thanks to J. Schubert and C. Witt for help running participants. T.H. has previously published under the name Tobias Schicke. Address correspondence to T. Collins, Psychology of Perception Laboratory, Paris Descartes University and CNRS, 45 rue des Saints-Pères, 75006 Paris, France (e-mail: collins.th@gmail.com).

\section{REFERENCES}

Abel, L. A., Schmidt, D., Dell'Osso, L. F., \& Daroff, R. B. (1978). Saccadic system plasticity in humans. Annals of Neurology, 4, 313318.

Awater, H., Burr, D., Lappe, M., Morrone, M. C., \& Goldberg, M. E. (2005). Effect of saccadic adaptation on localization of visual targets. Journal of Neurophysiology, 93, 3605-3614. doi:10.1152/ jn.01013.2004

BAHCALL, D., \& Kowler, E. (1999). Illusory shifts in visual direction accompany adaptation of saccadic eye movements. Nature, 26, 864866.

BLAUERT, J. (1997). Spatial hearing: The psychophysics of human sound localization. Cambridge, MA: MIT Press.

Bridgeman, B., Hendry, D., \& Stark, L. (1975). Failure to detect displacement of the visual world during saccadic eye movements. Vision Research, 15, 719-722.

Bruno, A., \& Morrone, M. C. (2007). Influence of saccadic adaptation on spatial localization: Comparison of verbal and pointing reports. Journal of Vision, 7(5, Art. 16), 1-13. doi:10.1167/7.5.16

Burr, D., Morrone, M. C., \& Ross, J. (2001). Separate visual representations for perception and action revealed by saccadic eye movements. Current Biology, 11, 798-802.
Collins, T., Doré-Mazars, K., \& Lappe, M. (2007). Motor space structures perceptual space: Evidence from human saccadic adaptation. Brain Research, 1172, 32-39.

Collins, T., Rolfs, M., Deubel, H., \& Cavanagh, P. (2009). Postsaccadic location judgments reveal remapping of saccade targets to non-foveal locations. Journal of Vision, 9(5, Art. 29), 1-9.

Deubel, H. (2004). Localization of targets across saccades: Role of landmark objects. Visual Cognition, 11, 173-202.

Deubel, H., Bridgeman, B., \& SchneIder, W. X. (1998). Immediate postsaccadic information mediates space constancy. Vision Research, 38, 3147-3159.

Doré-Mazars, K., Vergilino-Perez, D., Collins, T., Bohacova, K., \& Beauvillain, C. (2006). The use of recurrent signals about adaptation for subsequent saccade programming depends on object structure. Brain Research, 1113, 153-162.

Frens, M. A., \& VAn Opstal, A. J. (1994). Transfer of short-term adaptation in saccadic eye movements. Experimental Brain Research, 100, 293-306.

Frens, M. A., \& Van Opstal, A. J. (1995). A quantitative study of auditory-evoked saccadic eye movements in two dimensions. Experimental Brain Research, 107, 103-117.

Gaymard, B., Rivaud-Péchoux, S., Yelnik, J., Pidoux, B., \& Ploner, C. J. (2001). Involvement of the cerebellar thalamus in human saccade adaptation. European Journal of Neuroscience, 14, 554-560.

Goossens, H. H., \& VAN Opstal, A. J. (1999). Influence of head position on the spatial representation of acoustic targets. Journal of Neuroscience, $\mathbf{8 1}, 2720-2736$.

Groh, J. M., Trause, A. S., Underhill, A. M., Clark, K. R., \& INATI, S. (2001). Eye position influences auditory responses in primate inferior colliculus. Neuron, 29, 509-518.

Hofman, P. M., \& VAN Opstal, A. J. (1998). Spectro-temporal factors in two-dimensional human sound localization. Journal of the Acoustical Society of America, 103, 2634-2648.

Hopp, J. J., \& Fuchs, A. F. (2002). Investigating the site of human saccadic gain adaptation with targeting and express saccades. Experimental Brain Research, 144, 538-548.

HopP, J. J., \& Fuchs, A. F. (2004). The characteristics and neuronal substrate of saccadic eye movement plasticity. Progress in Neurobiology, 72, 27-53. doi:10.1016/j.pneurobio.2003.12.002

JAY, M. F., \& SPARKS, D. L. (1984). Auditory receptive fields in primate superior colliculus shift with changes in eye position. Nature, 309, 345-347.

JAY, M. F., \& Sparks, D. L. (1987a). Sensorimotor integration in the primate superior colliculus. I. Motor convergence. Journal of Neurophysiology, 57, 22-34.

JAY, M. F., \& SPARKS, D. L. (1987b). Sensorimotor integration in the primate superior colliculus. II. Coordinates of auditory signals. Journal of Neurophysiology, 57, 35-55.

Kommerell, G., Olivier, D., \& Theopold, H. (1976). Adaptive programming of phasic and tonic components in saccadic eye movements: Investigations in patients with abducens palsy. Investigative Ophthalmology, 15, 657-660.

KopinsKa, A., \& Harris, L. R. (2003). Spatial representation in body coordinates: Evidence from errors in remembering positions of visual and auditory targets after active eye, head, and body movements. $\mathrm{Ca}$ nadian Journal of Experimental Psychology, 57, 23-37.

LACKNER, J. R. (1973). The role of posture in sound localization. Quarterly Journal of Experimental Psychology, 26, 235-251.

LeWALD, J. (1997). Eye-position effects in directional hearing. Behavioural Brain Research, 87, 35-48.

LEWALD, J. (1998). The effect of gaze eccentricity on perceived sound direction and its relation to visual localization. Hearing Research, 115, 206-216.

LEWALD, J. (2002). Rapid adaptation to auditory-visual spatial disparity. Learning \& Memory, 9, 268-278.

Lewald, J., \& Ehrenstein, W. H. (1996a). Auditory-visual shift in localization depending on gaze direction. NeuroReport, 7, 1929-1932.

Lewald, J., \& Ehrenstein, W. H. (1996b). The effect of eye position on auditory lateralization. Experimental Brain Research, 108, 473-485.

Lewald, J., \& Ehrenstein, W. H. (2000). Visual and proprioceptive shifts in perceived egocentric direction induced by eye-position. $V i$ sion Research, 4, 539-547. 
MacAskill, M. R., Anderson, T. J., \& Jones, R. D. (2002). Adaptive modification of saccade amplitude in Parkinson's disease. Brain, 125, 1570-1582.

McLaughlin, S. (1967). Parametric adjustment in saccadic eye movements. Perception \& Psychophysics, 2, 359-362.

Melcher, D., \& Colby, C. L. (2008). Trans-saccadic perception. Trends in Cognitive Sciences, 12, 466-473.

Middlebrooks, J. C. (1992). Narrow-band sound localization related to external ear acoustics. Journal of the Acoustical Society of America, 92, 2607-2624.

Mullette-Gillman, O. A., Cohen, Y. E., \& Groh, J. M. (2005). Eyecentered, head-centered, and complex coding of visual and auditory targets in the intraparietal sulcus. Journal of Neurophysiology, 94, 2331-2352.

Mullette-Gillman, O. A., Cohen, Y. E., \& Groh, J. M. (2009). Motor-related signals in the intraparietal cortex encode locations in a hybrid, rather than eye-centered reference frame. Cerebral Cortex, 19, 1761-1775.

OldField, S. R., \& PARKer, S. P. (1984). Acuity of sound localisation: A topography of auditory space. II. Pinna cues absent. Perception, 13, 601-617.

Optican, L., \& Robinson, D. (1980). Cerebellar-dependent adaptive control of primate saccadic system. Journal of Neurophysiology, 44, 1058-1076.

Pavani, F., Husain, M., \& Driver, J. (2008). Eye-movements intervening between two successive sounds disrupt comparisons of auditory location. Experimental Brain Research, 189, 435-449.

Pavani, F., Husain, M., Làdavas, E., \& Driver, J. (2004). Auditory deficits in visuospatial neglect patients. Cortex, 40, 347-365.

Recanzone, G. H. (1998). Rapidly induced auditory plasticity: The ventriloquism aftereffect. Proceedings of the National Academy of Sciences, 95, 869-875.

Ross, J., Morrone, M. C., \& Burr, D. (1997). Compression of visual space before saccades. Nature, 386, 598-601.

Ross, J., Morrone, M. C., Goldberg, M., \& Burr, D. (2001). Changes in visual perception at the time of saccades. Trends in Neurosciences, 24, 113-121.

Russo, G. S., \& BRUCE, C. J. (1994). Frontal eye field activity preceding aurally guided saccades. Journal of Neurophysiology, 71, 1250-1253.

Sommer, M. A., \& Wurtz, R. H. (2008). Brain circuits for the internal monitoring of movements. Annual Review of Neuroscience, 31, 317338.

Stricanne, B., Andersen, R. A., \& Mazzoni, P. (1996). Eye-centered, head-centered, and intermediate coding of remembered sound locations in area LIP. Journal of Neurophysiology, 76, 2071-2076.

TAKeICHI, N., KANeKo, C. R. S., \& FuCHS, A. F. (2007). Activity changes in monkey superior colliculus during saccade adaptation. Journal of Neurophysiology, 97, 4096-4107.

Vliegen, J., Van Grootel, T. J., \& Van Opstal, A. J. (2004). Dynamic sound localization during rapid eye-head gaze shifts. Journal of Neuroscience, 24, 9291-9302.

Weerts, T. C., \& Thirlow, W. R. (1971). The effects of eye position and expectation on sound localization. Perception \& Psychophysics, 9, 35-39.

Werner-Reiss, U., Kelly, K. A., Trause, A. S., Underhill, A. M., \& GroH, J. M. (2003). Eye position affects activity in primary auditory cortex of primates. Current Biology, 13, 554-562.

(Manuscript received September 1, 2009; revision accepted for publication October 28, 2009.) 